\title{
Mobilisons-nous - Courons en faveur de la sécurité des patients!
}

\author{
Nadja Jennia , Olga Frank ${ }^{b}$, Christoph Bosshard ${ }^{\mathrm{c}}$, Esther Kraft ${ }^{\mathrm{d}}$ \\ ${ }^{a}$ M. Sc., collaboratrice scientifique FMH/ISFM, Division DDQ; ${ }^{b}$ Dr, responsable Projets/Produits/Prestations, Fondation Sécurité des Patients Suisse; \\ ${ }^{\circ}$ Dr, membre du Comité central de la FMH, responsable du département DDQ; dic. rer. oec., responsable de la division DDQ
}

La sécurité des patients est un thème qui nous concerne tous. Forte de ce constat, la fondation Sécurité des Patients Suisse a décidé de mettre sur pied une semaine d'action en collaboration avec ses partenaires, dont la FMH.

En Suisse, les activités visant à promouvoir la sécurité des patients sont nombreuses. Les médecins et les autres professionnels de la santé œuvrent sans relâche à améliorer la qualité des soins en s'engageant pour la sécurité des patients. Dans cette mission, ils peuvent compter sur le soutien de la fondation Sécurité des Patients Suisse qui a défini ce thème comme priorité et y travaille au niveau national.

La sécurité des patients est un thème qui nous concerne tous. Forte de ce constat, la fondation Sécurité des Patients Suisse a décidé de mettre sur pied une semaine d'action en collaboration avec ses partenaires, dont la FMH. Du 14 au 18 septembre 2015, sous le nom de "Semaine d'action pour la sécurité des patients - Dialogue avec les patients», cette action poursuit les objectifs suivants:

- Organiser des actions publiques au nom de la fondation et en collaboration avec les organisations partenaires et institutions de santé.

- Sensibiliser la population au thème de la sécurité des patients et à son importance en politique de la santé.

- Offrir aux institutions de santé une plateforme leur permettant de présenter les efforts qu'ils déploient en faveur de la sécurité des patients.

- Donner plus de visibilité à la fondation Sécurité des Patients Suisse au plan national et la positionner comme centre de compétences dans ce domaine.

Correspondance:

FMH

Nadja Jenni

Elfenstrasse 18

CH-3000 Berne 15

Tél. 0313591111

Fax 0313591112

nadja.jenni[at]fmh.ch
Suisse: un numéro spécial du Bulletin des médecins suisses paraîtra à cette occasion, et une course à pied visant à faire connaître les mesures contribuant à garantir la sécurité des soins sera organisée le 14 septembre 2015 à la Waisenhausplatz à Berne sous le slogan «Mobilisons-nous - Courons en faveur de la sécurité des patients!».

La FMH souhaite profiter de cette semaine d'action pour sensibiliser l'opinion publique à la sécurité des patients et faire connaître son propre engagement dans ce domaine.

Le financement de cette course à pied est assuré par une campagne de récolte de fonds qui permet à tous particuliers, fournisseurs de prestations, institutions, etc. - d'acheter des tours afin de courir eux-mêmes ou de parrainer la course. Les conditions de participation et de plus amples informations concernant la course sont disponibles sur le site internet de la FMH.

Chaque tour permet de sensibiliser davantage l'opinion publique à la problématique de la sécurité des patients et de récolter de l'argent pour la fondation Sécurité des Patients Suisse qui investira chaque centime en faveur des patients dans le cadre d'un projet ciblé. Organisé en collaboration avec la FMH, ce projet sera présenté par la fondation lors de la course. Vous aussi, mobilisez-vous en faveur de la sécurité des soins de santé de notre pays et soutenez la course à pied en achetant des tours jusqu'au 30 juin 2015. Vous trouverez les conditions de participation et de plus amples informations concernant la course sur le site internet de la FMH. 